Analysis and Application of High Resolution Numerical Perturbation Algorithm for ConvectiveDiffusion Equation

This article has been downloaded from IOPscience. Please scroll down to see the full text article.

2012 Chinese Phys. Lett. 29104702

(http://iopscience.iop.org/0256-307X/29/10/104702)

View the table of contents for this issue, or go to the journal homepage for more

Download details:

IP Address: 159.226.231.78

The article was downloaded on 04/12/2012 at 03:47

Please note that terms and conditions apply. 


\title{
Analysis and Application of High Resolution Numerical Perturbation Algorithm for Convective-Diffusion Equation *
}

\author{
GAO Zhi(高智), SHEN Yi-Qing(申义庆)** \\ LHD, Institute of Mechanics, Chinese Academy of Sciences, Beijing 100190
}

(Received 9 May 2012)

\begin{abstract}
The high resolution numerical perturbation (NP) algorithm is analyzed and tested using various convectivediffusion equations. The NP algorithm is constructed by splitting the second order central difference schemes of both convective and diffusion terms of the convective-diffusion equation into upstream and downstream parts, then the perturbation reconstruction functions of the convective coefficient are determined using the power-series of grid interval and eliminating the truncated errors of the modified differential equation. The important nature, i.e. the upwind dominance nature, which is the basis to ensuring that the NP schemes are stable and essentially oscillation free, is firstly presented and verified. Various numerical cases show that the NP schemes are efficient, robust, and more accurate than the original second order central scheme.
\end{abstract}

PACS: 47.11.Bc, 47.10.ad, 02.70.Bf

A number of numerical methods have been developed for computational fluid dynamics (CFD). The second order central difference $(2-\mathrm{CD})$ scheme has the comprehensive advantages of accuracy, efficiency, simplicity, and maintainability; hence it is regarded as a good scheme for some CFD applications, ${ }^{[1]}$ for example, it is used for the large eddy simulation in the pressure-based solver in FLUENT. However, the 2CD scheme generates spurious oscillations if the solution contains a large gradient or a discontinuity. How to overcome this drawback and improve its accuracy and robustness, without increasing any nodes and the complexity, is no doubt meaningful and practical. The numerical perturbation algorithm provides a new approach to achieve this outcome.

The numerical perturbation algorithm is to couple fluid dynamics effects with the discretized schemes of the convective diffusion equation. ${ }^{[2,3]}$ The main steps of constructing the algorithm are as follows: the flux and coefficient of the convective derivative in the mathematical basic schemes (the first order upwind scheme, the second order central schemes) are reconstructed as a power-series of grid intervals; using the convective-diffusion equation itself, the highorder fluid mechanics relation is obtained; by eliminating truncated error terms in the modified differential equation of the reconstructed scheme, the coefficients in the power-series are determined and finally the numerical perturbation algorithms are obtained.

Due to its physical preserving idea, and larger stable range and better accuracy than the original scheme, the numerical perturbation algorithm was applied to reconstruct various schemes,${ }^{[4-6]}$ and formed the high order perturbation difference scheme ${ }^{[2]}$ and perturbation finite volume scheme ${ }^{[3,7,8]}$ systems. Re- cently, based on the second order central difference scheme, $\mathrm{Gao}^{[9]}$ proposed a stable perturbation finite difference scheme (here it is called the high resolution numerical perturbation (NP) scheme) for the convective-diffusion equation. First, the second order difference schemes for both the convective and diffusion terms are split into two parts with upstream and downstream nodes, respectively, then the numerical perturbation is applied to reconstruct the split schemes. Numerical examples show that the new scheme is oscillation free even on coarse grids, and its errors are greatly less than the second order central scheme. In this Letter, the natures of the NP schemes are analyzed, and various numerical cases are calculated to verify the high performance of the NP schemes.

A general convective diffusion equation can be written as

$$
u \frac{\partial \phi}{\partial x}=\mu \frac{\partial^{2} \phi}{\partial x^{2}}
$$

where $u, \phi$ and $\mu$ denote the velocity, the transfer variable and the diffusion coefficient, respectively. If the second order central difference schemes are used to discretize both the convective and diffusion terms in Eq. (1), the discretized equation is written as

$$
\frac{u_{i}}{2 \Delta x}\left(\phi_{i+1}-\phi_{i-1}\right)=\frac{\mu}{\Delta x^{2}}\left(\phi_{i+1}-2 \phi_{i}+\phi_{i-1}\right) .
$$

As mentioned previously, the second order central difference scheme (2) generates an oscillation if the solution contains a large gradient or a discontinuity. The numerical perturbation algorithm aims at eliminating the non-physical numerical oscillation and obtaining a higher accurate solution. For completeness, the numerical perturbation algorithm ${ }^{[9]}$ is briefly introduced.

\footnotetext{
*Supported by the National Natural Science Foundation of China under Grant Nos 11272324 and 11272325.

** Correspondence author. Email: yqshen@imech.ac.cn

(C) 2012 Chinese Physical Society and IOP Publishing Ltd
} 
First, Eq. (2) is rewritten as

$$
\begin{aligned}
& {\left[\frac{u_{i}}{2 \Delta x}\left(\phi_{i}-\phi_{i-1}\right)+\frac{\mu}{\Delta x^{2}}\left(\phi_{i}-\phi_{i-1}\right)\right]} \\
& \quad+\left[\frac{u_{i}}{2 \Delta x}\left(\phi_{i+1}-\phi_{i}\right)-\frac{\mu}{\Delta x^{2}}\left(\phi_{i+1}-\phi_{i}\right)\right]=0 .
\end{aligned}
$$

The numerical perturbation scheme is written as

$$
\begin{aligned}
& {\left[\frac{u_{i} G_{i}^{+}}{2 \Delta x}\left(\phi_{i}-\phi_{i-1}\right)+\frac{\mu}{\Delta x^{2}}\left(\phi_{i}-\phi_{i-1}\right)\right]} \\
& \quad+\left[\frac{u_{i} G_{i}^{-}}{2 \Delta x}\left(\phi_{i+1}-\phi_{i}\right)-\frac{\mu}{\Delta x^{2}}\left(\phi_{i+1}-\phi_{i}\right)\right]=0
\end{aligned}
$$

The functions $G_{i}^{ \pm}$is the power series of grid interval $\Delta x$,

$$
G_{i}^{ \pm}=1+\sum_{n=1}^{N} a_{n}^{ \pm} \Delta x^{n} .
$$

Using the convective-diffusion equation (1) and freezing the coefficient of the convective term, we can obtain the approximate relation of high order derivatives of the transfer variable $\phi$ as follows:

$$
\frac{\partial^{n} \phi}{\partial x^{n}}=\left(\frac{u_{i}}{\mu}\right)^{n-1} \frac{\partial \phi}{\partial x} .
$$

We combined the Taylor series expansion and the method of undetermined coefficients (here, notice that the terms of $(\Delta x)^{-1}$ and $(\Delta x)^{0}$ are eliminated by the corrected Eq. (4) and the convective-diffusion equation (2), respectively; $G_{i}^{+}$and $G_{i}^{-}$are dependent on the first and the second parts of (4), respectively), coefficients $a_{n}^{ \pm}$are obtained as

$$
\begin{aligned}
& a_{2 n}^{ \pm}=0, \quad n=1,2, \ldots, \\
& a_{1}^{+}=-a_{1}^{-}=\frac{1}{3 !} \frac{u_{i}}{\mu}, \\
& a_{3}^{+}=-a_{3}^{-}=-\frac{1}{3 \times 5 !}\left(\frac{u_{i}}{\mu}\right)^{3}, \\
& a_{5}^{+}=-a_{5}^{-}=\frac{1}{3 \times 7 !}\left(\frac{u_{i}}{\mu}\right)^{5}, \\
& a_{7}^{+}=-a_{7}^{-}=-\frac{3}{5 \times 9 !}\left(\frac{u_{i}}{\mu}\right)^{7},
\end{aligned}
$$

Hence the $(2 N+1)$-th order (since $\left.a_{2 N}^{ \pm}=0\right)$ perturbation scheme is obtained by applying

$$
G^{ \pm}(N)=1+\sum_{n=1}^{2 N-1} a_{n}^{ \pm} \Delta x^{n}
$$

in Eq. (4).

In order to analyze the NP algorithm Eqs. (4) and (8) conveniently, the perturbation function Eq. (8) can be written as, for example,

$$
\begin{aligned}
G^{ \pm}(4)=1 & \pm \frac{1}{3 !} R_{\Delta x} \mp \frac{1}{3 \times 5 !} R_{\Delta x}^{3} \\
& \pm \frac{1}{3 \times 7 !} R_{\Delta x}^{5} \mp \frac{3}{5 \times 9 !} R_{\Delta x}^{7}
\end{aligned}
$$

where $R_{\Delta x}=\frac{u_{i} \Delta x}{\mu}$ is the Reynolds grid number.

The numerical perturbation scheme of Eqs. (4) and (9) has both mechanical and mathematical meanings:

(1) Equation (4) shows the clear upwind characteristic of the convective flow by splitting the spatial discretization into upstream and downstream parts.

(2) The perturbation functions $G^{ \pm}(N)$ of Eq. (9) are the polynomials of the Reynolds grid number, hence, the properties of NP schemes can be studied by using the Reynolds grid number.

(3) The spatial grid interval, which is the intrinsic small parameter in the numerical calculation, is used as the perturbation parameter to construct the numerical perturbation schemes.

(4) The accuracy of NP schemes is the discretization accuracy of the whole convective diffusion equation, while the accuracy of traditional difference schemes is usually the approximate accuracy of the first-order or second-order derivative in the equation.

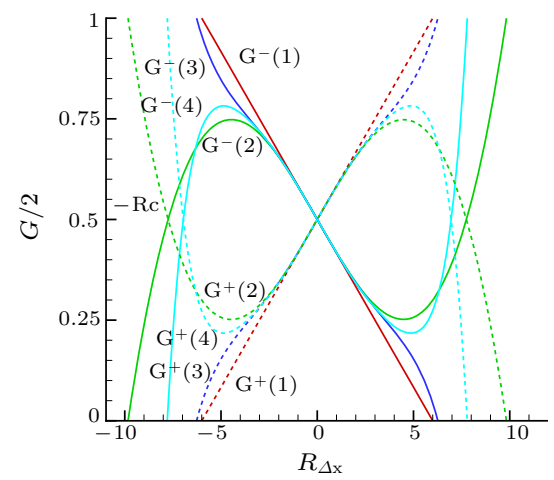

Fig. 1. Perturbation function vs Reynolds grid number.

By analyzing, the properties of the perturbation function $G^{ \pm}(N)$ are listed as follows:

(1) $\frac{1}{2}\left[G^{+}(N)+G^{-}(N)\right]=1$;

(2) $\left\{\begin{array}{l}G^{+}(1)>G^{-}(1), G^{+}(3)>G^{-}(3), \text { if } u_{i}>0, \\ G^{-}(1)>G^{+}(1), G^{-}(3)>G^{+}(3), \text { if } u_{i}<0\end{array}\right.$

(3) $\left\{\begin{array}{l}G^{+}(2)>G^{-}(2), \text { if } 0<R_{\Delta x}<R c\left(u_{i}>0\right), \\ G^{-}(2)>G^{+}(2), \text { if } 0>R_{\Delta x}>-R c\left(u_{i}<0\right)\end{array}\right.$

Here $G^{ \pm}(4)$ has the same property as $G^{ \pm}(2)$, except that the $R c$ is different. $R c$ can be regarded as a critical grid Reynolds number, which is defined as a positive minimum value to make $G^{+}(N)=G^{-}(N)$. For example, $R c=\sqrt{60}$ is solved for $G^{ \pm}(2)$.

Figure 1 shows the distribution of the perturbation function $G^{ \pm}(N)$ vs the Reynolds grid number 
$R_{\Delta x}$. The above properties indicate that the thirdand seventh-order NP schemes are upwind dominant, the fifth- and ninth-order NP schemes are conditionally upwind dominant in a proper range of $R_{\Delta x}$. The property of upwind dominance is the essential nature of the NP schemes, hence the NP schemes are essentially oscillation free. If $G^{ \pm}(N) \geq 0$ is required, the form of NP schemes looks like the weighted scheme of the first order upstream and downstream schemes.

Several cases are calculated to demonstrate the efficiency, robustness and high order accuracy of the NP schemes. In this study, the time dependent method is applied to obtain the steady solution. The fourth order Runge-Kutta method is used for the time marching.

Table 1. The linear convective diffusion equation for $R e=$ 1000.

\begin{tabular}{cccccc}
\hline Scheme & $N$ & $L_{\infty}$ error & $L_{\infty}$ order & $L_{1}$ error & $L_{1}$ order \\
\hline 2-CD & 80 & OS & & & \\
& 160 & OS & & & \\
& 320 & OS & & & \\
& 640 & $0.8629 \times 10^{-1}$ & & $0.1948 \times 10^{-3}$ & \\
\hline 3 -NP & 80 & 0.3743 & & $0.7386 \times 10^{-2}$ & \\
& 160 & 0.1479 & 1.34 & $0.1083 \times 10^{-2}$ & 2.77 \\
& 320 & $0.2996 \times 10^{-1}$ & 2.30 & $0.1056 \times 10^{-3}$ & 3.36 \\
& 640 & $0.3080 \times 10^{-2}$ & 3.28 & $0.7742 \times 10^{-5}$ & 3.77 \\
\hline $5-\mathrm{NP}$ & 80 & OV & & & \\
& 160 & OS & & & \\
& 320 & $0.7078 \times 10^{-2}$ & & $0.2399 \times 10^{-4}$ & \\
& 640 & $0.1782 \times 10^{-3}$ & 5.31 & $0.4460 \times 10^{-6}$ & 5.75 \\
\hline $7-\mathrm{NP}$ & 80 & 0.8750 & & $0.8639 \times 10^{-1}$ & \\
& 160 & 0.1254 & 2.80 & $0.8945 \times 10^{-3}$ & 6.59 \\
& 320 & $0.1687 \times 10^{-2}$ & 6.22 & $0.5771 \times 10^{-5}$ & 7.28 \\
& 640 & $0.1080 \times 10^{-4}$ & 7.29 & $0.2704 \times 10^{-7}$ & 7.74 \\
\hline $9-\mathrm{NP}$ & 80 & OV & & & \\
& 160 & OS & & & \\
& 320 & $0.4116 \times 10^{-3}$ & & $0.1405 \times 10^{-5}$ & \\
& 640 & $0.6611 \times 10^{-6}$ & 9.28 & $0.1656 \times 10^{-8}$ & 9.73 \\
\hline
\end{tabular}

The linear convective diffusion equation is used as the first test case and can be written as

$$
\frac{\partial u}{\partial t}+\frac{\partial u}{\partial x}=\frac{1}{R e} \frac{\partial^{2} u}{\partial x^{2}}, \quad 0 \leq x \leq 1 .
$$

The steady state solution is $u(x)=\left(e^{R e x}-1\right) /\left(e^{R e}-\right.$ $1)$. The comparison of the third order NP (3-NP) algorithm and the second order central difference scheme with grid number $N=160$ is shown in Fig. 2. Near the large gradient, the second order central scheme causes dramatic oscillations. The 3-NP scheme is oscillation free and agrees well with the exact solution. Table 1 shows the detailed error comparison of different schemes. In this study, OS denotes the oscillatory solution, OV denotes the overflow happened in the calculation. It can be seen that, if a large grid interval (means large Reynolds grid number) is used, the second order central scheme is oscillatory. If $N=80$ is used, the $R_{\Delta x}$ equals 12.5 , which is larger than $R_{c}$ of $G(2)$ and $G(4)$, hence the 5-NP and 9-NP schemes are overflow. If $N=160$ is used, the $R_{\Delta x}$ equals to 6.25 , which is close to $R_{c}$, the 5 -NP and 9-NP schemes are weak upwind dominant and result in a small oscillation near the large gradient region. Meanwhile, with the large Reynolds grid number (for example, $N=80$ ), the 7-NP scheme is not necessarily more accurate than the 3-NP scheme. With the grid number increased, the NP schemes achieve the expected order, for example, the third order, fifth order, seventh order, and ninth order, respectively.

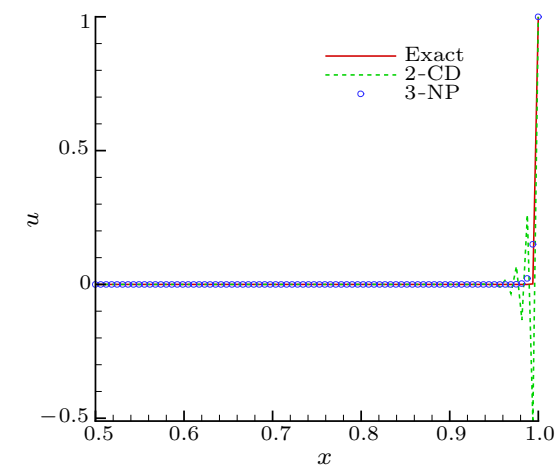

Fig. 2. The linear convective diffusion equation for $R e=$ 1000 .

Table 2. The nonlinear Burgers equation, $R e=10$.

\begin{tabular}{cccccc}
\hline Scheme & $N$ & $L_{\infty}$ error & $L_{\infty}$ order & $L_{1}$ error & $L_{1}$ order \\
\hline 2-CD & 80 & $0.2223 \times 10^{-2}$ & & $0.6919 \times 10^{-3}$ & \\
& 160 & $0.5667 \times 10^{-3}$ & 1.97 & $0.1756 \times 10^{-3}$ & 1.98 \\
& 320 & $0.1427 \times 10^{-3}$ & 1.99 & $0.4404 \times 10^{-4}$ & 2.00 \\
& 640 & $0.3517 \times 10^{-4}$ & 2.02 & $0.1080 \times 10^{-4}$ & 2.03 \\
\hline 3 -NP & 80 & $0.9514 \times 10^{-3}$ & & $0.2498 \times 10^{-3}$ & \\
& 160 & $0.2434 \times 10^{-3}$ & 1.97 & $0.6341 \times 10^{-4}$ & 1.98 \\
& 320 & $0.6093 \times 10^{-4}$ & 2.00 & $0.1575 \times 10^{-4}$ & 2.01 \\
& 640 & $0.1463 \times 10^{-4}$ & 2.06 & $0.3696 \times 10^{-5}$ & 2.09 \\
\hline 7 -NP & 80 & $0.9522 \times 10^{-3}$ & & $0.2501 \times 10^{-3}$ & \\
& 160 & $0.2434 \times 10^{-3}$ & 1.97 & $0.6343 \times 10^{-4}$ & 1.98 \\
& 320 & $0.6093 \times 10^{-4}$ & 2.00 & $0.1575 \times 10^{-4}$ & 2.01 \\
& 640 & $0.1463 \times 10^{-4}$ & 2.06 & $0.3696 \times 10^{-5}$ & 2.09 \\
\hline
\end{tabular}

Table 3. The nonlinear Burgers equation for $R e=1000$.

\begin{tabular}{cccccc}
\hline \multirow{2}{*}{$N$} & 2 -CD & \multicolumn{2}{c}{3 -NP } & \multicolumn{2}{c}{7 -NP } \\
\cline { 2 - 6 } & \multicolumn{1}{c}{$L_{\infty}$} & $L_{1}$ & $L_{\infty}$ & $L_{1}$ \\
\hline 80 & OS & 0.4688 & $0.2720 \times 10^{-1}$ & 0.7099 & 0.2713 \\
160 & OS & 0.2848 & $0.5511 \times 10^{-2}$ & 0.4374 & $0.2872 \times 10^{-1}$ \\
320 & OS & 0.1200 & $0.8816 \times 10^{-3}$ & $0.8439 \times 10^{-1}$ & $0.6077 \times 10^{-3}$ \\
640 & OS & $0.4734 \times 10^{-2}$ & $0.2220 \times 10^{-4}$ & $0.3154 \times 10^{-1}$ & $0.1031 \times 10^{-3}$ \\
\hline
\end{tabular}

The nonlinear Burgers equation is the second test case. It is written as

$$
\begin{gathered}
\frac{\partial u}{\partial t}+u \frac{\partial u}{\partial x}=\frac{1}{R e} \frac{\partial^{2} u}{\partial x^{2}}, \quad a \leq x \leq b, \\
u(a)=\tanh (-a R e / 2), \quad u(b)=\tanh (-b R e / 2) .
\end{gathered}
$$

The steady state solution of Eq. (10) with boundary condition (11) is $u(x)=\tanh (-x R e / 2)$. At $x=0$, the shock is formed with a large Re number. In our computation, $a=-1, b=1$ are used. Based on the analysis of the 5 th-order and 9th-order NP schemes, which 
are conditionally upwind dominant, and the numerical results of previous cases, only the 3rd-order and the 7 th-order schemes are considered for this case.

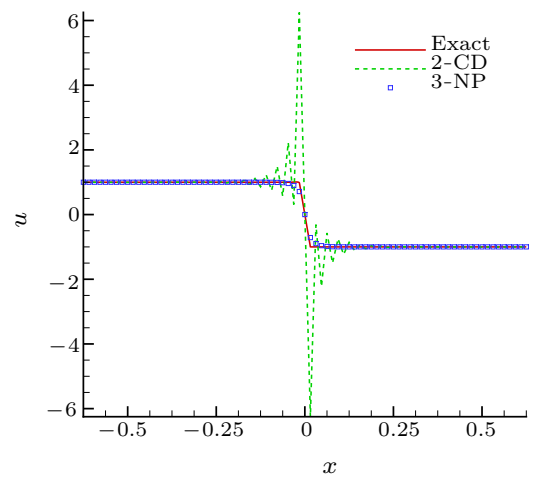

Fig. 3. Nonlinear Burgers equation for $R e=1000$.

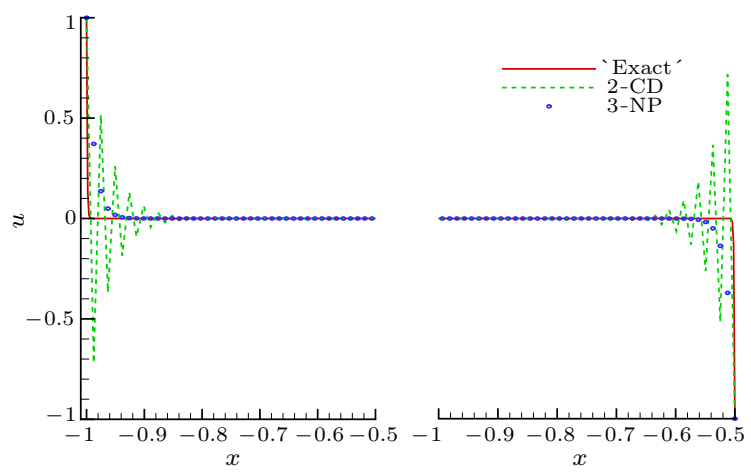

Fig. 4. Variable coefficients convective diffusion equation for $R e=1000$.

First, the case with $R e=10$ is tested. Table 2 gives the errors. Since the small Reynolds number is used, all tested schemes obtain good results. It can also be seen that the errors of the NP schemes are no more than one half of the second central scheme. Then, the case with $R e=1000$ is calculated, and the errors are given in Table 3 . The second order central scheme is oscillatory even with the finest grid number of $N=640$. The NP schemes are oscillation free. Figure 3 gives the comparison of the second order central scheme and the third order NP scheme with $R e=1000$ and $N=160$.

It is worth mentioning that, for this nonlinear case, the relation of high order derivatives (6) is approximate, hence the results of the high order (7th-order) NP scheme are almost the same as the low order (3rdorder) NP scheme. Hence, for the other cases in this paper, only the 2-CD scheme and the 3 -NP scheme are compared.

The variable coefficients convective diffusion equation, i.e.

$$
\frac{\partial u}{\partial t}+x \frac{\partial u}{\partial x}=\frac{1}{R e} \frac{\partial^{2} u}{\partial x^{2}}, \quad-1 \leq x \leq 1
$$

under the boundary conditions

$$
u(-1)=1, \quad u(1)=-1,
$$

is the third test case. The comparison of the steady state solution with $R e=1000$ and $N=160$ is given in Fig. 4. Here the exact solution is the result calculated by the third order NP scheme with $N=1000$. The second central scheme is still oscillatory, while the third order NP scheme obtains a good solution.

The convective diffusion equation with source terms is the fourth test case,

$$
\begin{aligned}
\frac{\partial u}{\partial t}+\frac{\partial u}{\partial x}= & \frac{1}{R e} \frac{\partial^{2} u}{\partial x^{2}}+\epsilon \pi^{2} \sin (\pi x)+\pi \cos (\pi x), \\
& 0 \leq x \leq 1
\end{aligned}
$$

The exact solution is given by $u(x)=\sin (\pi x)+\left(e^{x / \epsilon}-\right.$ $1) /\left(e^{1 / \epsilon}-1\right)$, where $\epsilon=1 / R e$. Table 4 gives the comparison of errors. The 2-CD scheme is oscillatory, even the mesh of $N=320$ is used. The $3-\mathrm{NP}$ scheme is oscillation free, and it reaches the second order for both $L_{\infty}$ and $L_{1}$ norms. Figure 5 plots the results of $R e=1000$ and $N=320$. This case shows that the NP scheme can also solve the convective diffusion equation with source terms very well.

Table 4. The convective diffusion equation with source terms for $R e=1000$.

\begin{tabular}{cccccc}
\hline Scheme & $\mathrm{N}$ & $L_{\infty}$ error & $L_{\infty}$ order & $L_{1}$ error & $L_{1}$ order \\
\hline 2-CD & 80 & $0.7182(\mathrm{OS})$ & & $0.3158 \times 10^{-1}$ & \\
& 160 & $0.5127(\mathrm{OS})$ & 0.49 & $0.6531 \times 10^{-2}$ & 2.27 \\
& 320 & $0.2613(\mathrm{OS})$ & 0.97 & $0.1005 \times 10^{-2}$ & 2.70 \\
& 640 & $0.8629 \times 10^{-1}$ & 1.60 & $0.1973 \times 10^{-3}$ & 2.35 \\
\hline 3-NP & 80 & 0.3256 & & $0.4167 \times 10^{-1}$ & \\
& 160 & 0.1309 & 1.32 & $0.1058 \times 10^{-1}$ & 1.98 \\
& 320 & $0.2529 \times 10^{-1}$ & 2.37 & $0.2575 \times 10^{-2}$ & 2.04 \\
& 640 & $0.2081 \times 10^{-2}$ & 3.60 & $0.6327 \times 10^{-3}$ & 2.03 \\
\hline
\end{tabular}

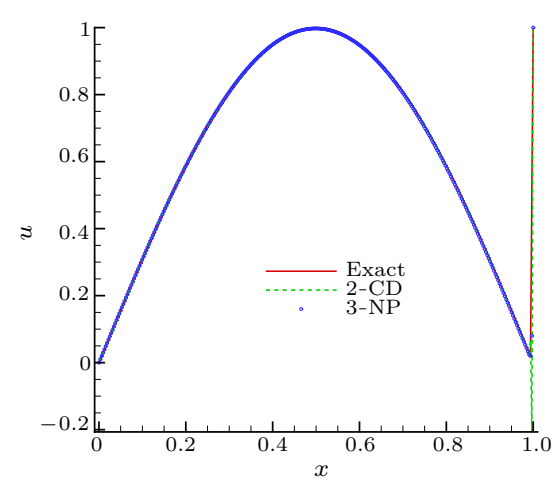

Fig. 5. Convective diffusion equation with source terms for $R e=1000$.

The two-dimensional nonlinear Burgers equation is the fifth test case,

$$
\begin{aligned}
\frac{\partial u}{\partial t}+u \frac{\partial u}{\partial x}+u \frac{\partial u}{\partial y}= & \frac{1}{R e}\left(\frac{\partial^{2} u}{\partial x^{2}}+\frac{\partial^{2} u}{\partial y^{2}}\right) \\
& -1 \leq x, y \leq 1
\end{aligned}
$$


For the two-dimensional convective diffusion equation, combined with the spatial splitting method, the numerical perturbation procedure of Eqs. (4) and (9) is applied in each spatial direction with its corresponding Reynolds grid number. The exact solution is given by $u(x, y)=\tanh (-\operatorname{Re}(x+y) / 2)$. The second order central scheme is overflow even the grid of $320 \times 320$ is used. Table 5 gives the errors of the 3 -NP scheme. Figure 6 is the solution obtained by the 3 -NP scheme with the grid of $160 \times 160$.

Table 5. Two-dimensional nonlinear Burgers equation for $R e=1000$.

\begin{tabular}{|c|c|c|c|c|}
\hline \multirow[t]{2}{*}{$N_{x} \times N_{y}$} & \multicolumn{2}{|c|}{ 2-CD } & \multicolumn{2}{|r|}{ 3-NP } \\
\hline & $L_{\infty}$ & $L_{1}$ & $L_{\infty}$ & $L_{1}$ \\
\hline $40 \times 40$ & $\mathrm{OV}$ & & 0.6420 & 0.1434 \\
\hline $80 \times 80$ & $\mathrm{OV}$ & & 0.4688 & $0.2597 \times 10^{-1}$ \\
\hline $160 \times 160$ & OV & & 0.2848 & $0.5440 \times 10^{-2}$ \\
\hline $320 \times 320$ & OV & & 0.1213 & $0.8887 \times 10^{-3}$ \\
\hline
\end{tabular}

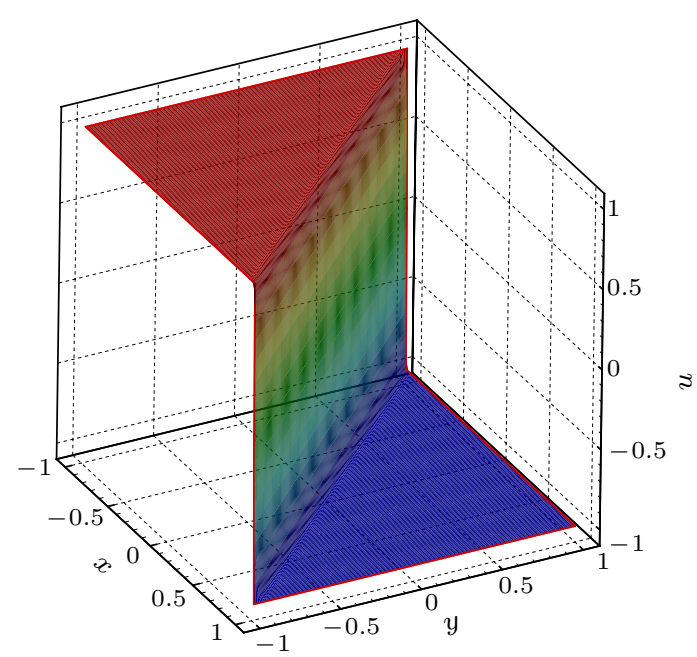

Fig. 6. Two-dimensional nonlinear Burgers equation for $R e=1000$ in the 3 -NP scheme.

Finally, the two-dimensional linear convective diffusion equation with variable coefficients is calculated,

$$
\begin{aligned}
\frac{\partial u}{\partial t}+\left(y-\frac{1}{2}\right) \frac{\partial u}{\partial x}+\left(x-\frac{1}{2}\right) \frac{\partial u}{\partial y}= & \frac{1}{R e}\left(\frac{\partial^{2} u}{\partial x^{2}}+\frac{\partial^{2} u}{\partial y^{2}}\right) \\
& -1 \leq x, y \leq 1
\end{aligned}
$$

The exact solution is given by $u(x, y)=\exp [\operatorname{Re}(x-$ $\left.\left.\frac{1}{2}\right)\left(y-\frac{1}{2}\right)\right]$. The case with $R e=40$ is calculated. Since the Reynolds grid number is small relatively, the 2CD scheme and the 3 -NP scheme can run in this case. However, the errors given in Table 6 show that the second central scheme gives unacceptable results even with the mesh of $160 \times 160$, while the third order NP scheme obtains a very good solution and it achieves third order accuracy. Figure 7 is the solution calculated by the 3 -NP scheme with mesh of $80 \times 80$.

Table 6. Two-dimensional convective diffusion equation with variable coefficients for $R e=40$.

\begin{tabular}{ccccc}
\hline \multirow{2}{*}{$N_{x} \times N_{y}$} & \multicolumn{2}{c}{$2-\mathrm{CD}$} & \multicolumn{2}{c}{$3-\mathrm{NP}$} \\
\cline { 2 - 5 } & $L_{\infty}$ & $L_{1}$ & $L_{\infty}$ & $L_{1}$ \\
\hline $40 \times 40$ & $0.3740 \times 10^{2}$ & $0.1028 \times 10^{1}$ & 0.1199 & $0.2856 \times 10^{-2}$ \\
$80 \times 80$ & $0.1077 \times 10^{2}$ & 0.2758 & $0.8903 \times 10^{-2}$ & $0.2022 \times 10^{-3}$ \\
$160 \times 160$ & $0.2774 \times 10^{1}$ & $0.7072 \times 10^{-1}$ & $0.5997 \times 10^{-3}$ & $0.1354 \times 10^{-4}$ \\
\hline
\end{tabular}

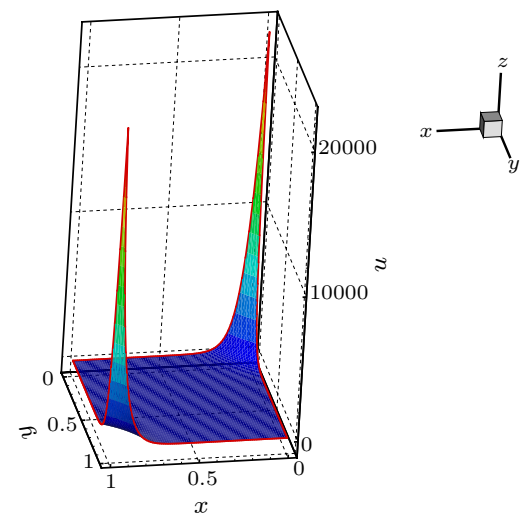

Fig. 7. Two-dimensional convective diffusion equation with variable coefficients for $R e=40$ in the $3-\mathrm{NP}$ scheme.

In summary, the nature of upwind dominance or conditionally upwind dominance of the NP schemes is presented and verified. It is this nature that makes the NP schemes essentially non-oscillatory schemes in the large gradient region or near discontinuity even with a large Reynolds grid number. Various numerical examples show that the NP schemes are not only efficient and robust, but also more accurate than the original second order central scheme. The application to fluid dynamics Navier-Stokes equations and the multi-nodes NP reconstruction algorithm are currently underway and will be reported elsewhere.

\section{References}

[1] Fletcher C A J 1997 Computational Techniques for Fluid Dynamics (Berlin: Springer)

[2] Gao Z 2000 Adv. Mech. 30200 (in Chinese)

[3] Gao Z 2010 Adv. Mech. 40607 (in Chinese)

[4] Shen Y Q, Gao Z and Yang D H 2003 ACTA Aerodynamica Sin. 21342 (in Chinese)

[5] Yang M Y, Shu S and Li M J 2010 Chin. J. Hydrodyn. 25 307 (in Chinese)

[6] Zhu K and Li M J 2011 Chin. J. Theor. Appl. Mech. 4355 (in Chinese)

[7] Gao Z and Yang G W 2004 Acta Mech. Sin. 20580

[8] Gao Z, Shen Y Q and Yang G W 2004 CFD J. 13204

[9] Gao Z 2010 Chin. J. Theor. Appl. Mech. 42811 (in Chinese) 